\title{
Über eine neue aus dem Pyridin erhaltene Base
}

\author{
von
}

Franz v. Hemmelmayr.

Aus dem chemischen Laboratorium der k. k. deutschen Universität in Prag.

(Vorgelegt in der Sitzung am 16. Juli 1891.)

Vor nicht ganz einem Jahre erhielt Lösekann ${ }^{1}$ durch Einwirkung von Chlorwasserstoff auf Formaldehyd einen eigenthïmlichen Körper, dem er den Namen Chlormethylalkohol beilegte und der auch einige Zeit von der Firma "Merklin und Lösekann" in Hannover in den Handel gebracht wurde. Diese Verbindung, die in ihrem Verhalten grosse Ähnlichkeit mit den Alkylhalogenen hat, ist wie dies Lösekann auch gleich nach seiner Entdeckung aussprach, ungemein reactionsfähig, besonders solchen Verbindungen gegenüber, die Alkohol- oder Ammoniakreste enthalten.

Wie ich bereits früher ${ }^{2}$ einmal mitgetheilt habe, entstehen bei der Einwirkung von Chlormethyalkohol auf Harnstoffe die Methylen-Derivate derselben.

Es erschien mir nun einladend zu untersuchen, wie sich stickstoffhältige Kerne gegenüber dem Chlormethylalkohol verhalten würden und habe ich dieses Mal das Pyridin zum Ausgangspunkte der Untersuchung gewählt und ist es mir gelungen, ein Additionsprodukt desselben zu erhalten.

Im Folgenden sei nun ein Bild des Reactionsverlaufes gegeben, woran ich eine Beschreibung des entstandenen Productes schliessen will.

1 Chem. Zeit., 14; 1408-1409.

2 Monatshefte f. Chemie, XII, 89 
Pyridin und Chlormethylalkohol wirken sehr heftig aufeinander ein, so dass es bei raschem Eingiessen des letzteren sehr leicht vorkommen kann, dass ein Theil der Flüssigkeit aus dem Gefässe geschleudert wird.

Es ist desshalb nothwendig, durch sorgfältiges Kühlen die Reaction zu mässigen.

Schon beim Zusammenbringen mit den ersten Tropfen Chlormethylalkohol scheidet sich eine röthlichbraun gefärbte, dicke, ölige Flüssigkeit aus, die mit der zugesetzten Menge wächst, bis schliesslich die ganze Masse sich in einen dicken, dunkelroth gefärbten Syrup verwandelt hat, womit das Ende der Reaction erreicht ist. Es tritt dies ein, wenn man beiläufig das gleiche Gewicht Chlormethylalkohol zum Pyridin hinzugefügt hat.

Lässt man diesen Syrup längere Zeit in der Kälte stehen, so wird er krystallinisch, es gelingt jedoch nicht, die Krystalle zu isoliren und durch Umkrystallisiren zu reinigen, da sie ungemein hygroskopisch sind und bei längerem Stehen bei Zimmertemperatur in halbwegs feuchter Luft wieder zerfliessen.

Zur Reinigung wurde das Reactionsproduct desshalb mit Alkohol vorerst etwas abgespült, hierauf in demselben gelöst, die Lösung abgedampft und der zurückbleibende Syrup in Wasser aufgenommen.

Aus dieser Lösung, liessen sich durch eine Reibe von Salzen, wie auch von Säuren scbön krystallisirte Verbindungen ausscheiden, die zur Charakterisirung des entstandenen Körpers dieuten.

Einige von ilnen will ich nachstehend bescbreiben.

1. Doppelverbindung mit Quecksilbercblorid.

Fügt man zu der oben erwähnten wässerigen Lösung Sublimatlösung hinzu, so entsteht sofort ein weisser, dichter Niederschlag, der sehr bald in die krystallinische Form iibergeht.

Löst man diesen Niedersehlag in heissem Wasser, so scheiden sich beim Erkalten glänzende, schwach röthlich gefärbte Blättchen ab, die auf der Saugpumpe filtrirt und mit Alkohol und Äther gut gewaschen wurden.

Bei der Analyse ergaben sich folgende Werthe:

I. $0 \cdot 4715 g$ Substanz gaben $0 \cdot 2642 g \mathrm{Hg}$, entsprechend $0 \cdot 22775 \mathrm{~g} \mathrm{Hg}$ oder $48 \cdot 30 \% \mathrm{Hg}$. 
II. $0.5435 \mathrm{~g}$ Substanz gaben $0.3040 \mathrm{~g} \mathrm{Hg}$, entsprechend $0 \cdot 26207 \mathrm{~g} \mathrm{Hg}$ oder $48 \cdot 21 \% \mathrm{Hg}$.

Nehmen wir nun an, es habe in der That eine Anlagęrung. von Chlormethylalkohol an das Pyridin stattgefunden, so hätte dieser Körper offenbar die Formel:

$$
\mathrm{C}_{5} \mathrm{H}_{\mathfrak{s}} \mathrm{N} . \mathrm{CH}_{2} \mathrm{OH} . \mathrm{Cl}
$$

und die Formel der Doppelverbindung mit dem Sublimat müsste im einfachsten Falle sein:

$$
\mathrm{C}_{5} \mathrm{H}_{5} \mathrm{~N} \cdot \mathrm{CH}_{2} \cdot \mathrm{OHCl} \cdot \mathrm{HgCl}_{2} \text {. }
$$

Mit dieser Formel stehen auch wirklich die obigen Analysen im Einklang.

Denn es sind in 100 Theilen:

I.

$\mathrm{Hg} . .48 \cdot 30$
II.
Theorie für

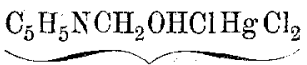

$48 \cdot 02$.

Diese Doppelverbindung mit Sublimat ist in kaltem Wasser schwer, in heissem aber ziemlich leicht löslich. Der Schmelzpunkt liegt bei $162^{\circ} \mathrm{C}$.

2. Doppelverbindung mit Platinchlorid.

Auf Zusatz von Platinchlorid zur wässerigen Lösung des Reactionsproductes erhält man einen gelben Niederschlag, der sich aus heissem Wasser gut umkrystallisiren lässt.

Eine Analyse lieferte folgenden Werth: $0 \cdot 2974 g$ Substanz gaben $0.0915 g$ Pt.

Demnach sind in 100 Theilen:

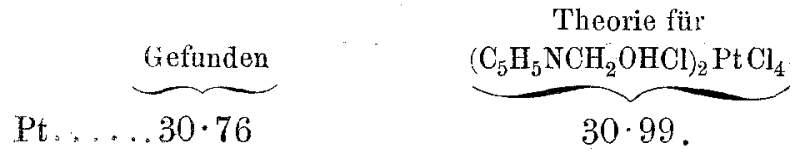

Das Platindoppelsalz bildet schön ausgebildete, gelbe, prismatische Krystalle, die sich in kaltem Wasser schwer, in heissem aber ziemlich leicht lösen. Der Schmelzpunkt liegt bei $216^{\circ} \mathrm{C}$. 
F. v. Hemmelmayr,

\section{Verbindung mit Pikrinsäure.}

Eine kalt gesättigte Lösung von Pikrinsäure wurde zur wässerigen Lösung der chlorwasserstoffsauren Verbindung hinzugegeben. Es entstand ein gelber Niederschlag, der filtrirt, gewaschen und in heissem Wasser gelöst wurde. Beim Erkalten schieden sich sehr schöne lange Prismen aus, die nochmals umkrystallisirt und dann zur Analyse verwendet wurden.

I. $0.523 \mathrm{~g}$ Substanz lieferten $0.1454 \mathrm{~g} \mathrm{H}_{2} \mathrm{O}$ und $0.821 g \mathrm{CO}_{2}$, entsprechend $0 \cdot 01616 \mathrm{~g} \mathrm{H}$ und $0 \cdot 2239 \mathrm{~g} \mathrm{C}$.

II. $0 \cdot 3308 \mathrm{~g}$ Substanz gaben $51 \mathrm{~cm}^{3}$ feuchten Stickstoff bei $24^{\circ} \mathrm{C}$. und $746 \mathrm{~mm}$ Barometerstand, entsprechend $0.056105 \mathrm{~g} \mathrm{~N}$.

Es sind demnach in 100 Theilen:

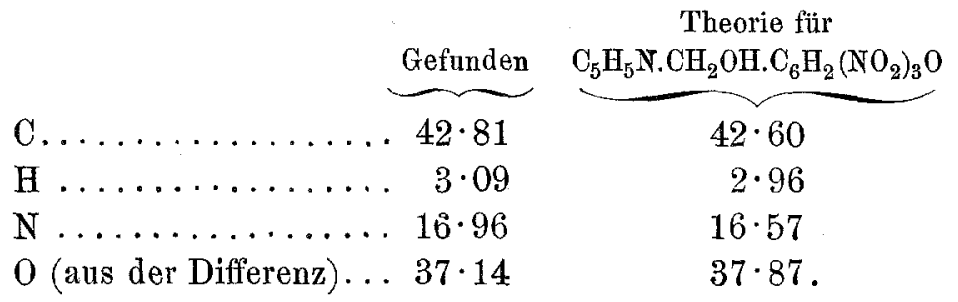

Der Schmelzpunkt des Pikrates liegt bei beiläufig $200^{\circ} \mathrm{C}$.

4. Verbindung mit Salpetersäure.

Zur Herstellung dieser Verbindung wurde zur salzsanren Base, wie sie aus dem Chlormethylalkohol und dem Pyridin erhalten worden, solange Silbernitratlösung hinzugefügt, bis die Salzsäure genau ausgefällt war.

Die vom Chlorsilber abfiltrirte Fltissigkeit wurde auf dem Wasserbade eingedampft, der Riickstand in Alkohol gelöst und zur so erhaltenen alkoholischen Lösung Äther im Überschuss zugesetzt. Die Flüssigkeit trübte sich anfangs milehig, bald aber schieden sich farblose, gut ansgebildete, prismatische Krystalle aus.

Eine mit diesen Krystallen vorgenommene Stickstoff bestimmung lieferte folgendes Resultat:

$0.5565 \mathrm{~g}$ Substanz gaben $85 \mathrm{~cm}^{3}$ feuchten Stickstoff bei $23^{\circ} \mathrm{C}$.

und $735 \mathrm{~mm}$ Barometerstand, entsprechend $0.09256 \mathrm{~g} \mathrm{~N}$. 
Demnach enthalten 100 Theile:

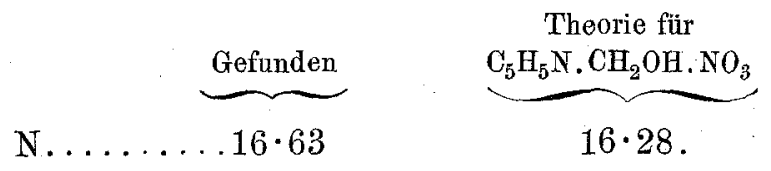

5. Verbindung mit Schwefelsäure.

Eine Verbindung mit Schwefelsäure konnte nicht in Krystallgestalt erhalten werden. Es wurde zwar in analoger Weise wie beim Nitrat vorgegangen, allein auch durch Zusatz von Äther schied sich die Verbindung bloss in Form eines Syrups aus.

Ebenso resultatlos war ein Versuch, den Syrup durch Stehen im Vacuum über concentrirter Schwefelsäure zum Krystallisiren zu bringen.

Aus den analytischen Resultaten aller dieser Verbindungen geht wohl hervor, dass der Körper, der beim Zusammenbringen von Pyridin mit Chlormethylalkohol entsteht, das durch directe Addition gebildete Chlorid einer quaternären Base ist.

Es ist mir zwar nicht gelungen, dieses chlorwasserstoffsanre Salz derart zu isoliren, respective so rein zu erhalten, wie es zu einer Analyse nöthig gewesen wäre, allein die durchaus sehr schön krystallisirenden Doppelverbindungen mit Sublimat, Platinchlorid etc. beweisen zur Genüge, dass es wirklich existirt. Die Formel desselben wäre dann, wie bereits früher erwähnt wurde:

$$
\mathrm{C}_{5} \mathrm{H}_{5} \mathrm{~N} . \mathrm{CH}_{2} \mathrm{OH} . \mathrm{Cl} \text {. }
$$

Um die freie Base selbst darzustellen, wurde zur salzsauren Verbindung in wässeriger Lösung frisch bereitetes Silberoxyd gegeben und hierauf längere Zeit damit geschüttelt.

Die Flüssigkeit färbte sich dabei braun, und gleichzeitig. trat der intensive Geruch des Pyridins auf, während die Wände des Gefässes sich mit einem Silberspiegel überzogen, der besonders beim längeren Stehen deutlich hervortrat. Es war also offenbar Zersetzung in die Componenten eingetreten. 
Dies zeigte sich noch deutlicher, als eine filtrirte Probe im Vacuum verdunstet warde. Es blieb nichts zurück als ein Beschlag von reducirtem Silber, herribhrend von der Reduction des in Wasser etwas löslichen Silberoxydes durch den gebildeten Formaldehyd. Auch Kalilauge zersetzt das salzsaure Salz unter Braunfärbung in Formaldehyd und Pyridin. Jedenfalls geht die Zersetzung aber theilweise noch weiter, da sonst die Dunkelfärbung nicht recht gut za erklären wäre. Eine Isolirung der freien Base gelang sonach nicht.

Die Constitution der salzsauren Base, die ja doch zuerst erhalten wird, glaube ich mir bloss in der Weise denken zu köunen, dass sich der Chlormethylalkohol an den Stickstoff des Pyridins anlagert, indem derselbe fünfwerthig wird. Man hat dann folgendes Formelbild:

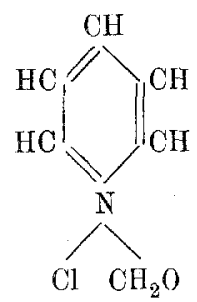

Wird nun das Chlor durch das Silber herausgeholt, so wird Formaldehyd und Pyridin regenerirt. Man könnte sich die Constitution der Base dann nur so vorstellen:

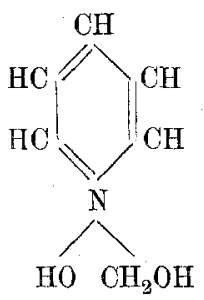

und eine solche Verbindung müsste, wenn sie überhaupt existenzfähig wäre, doch nur eine sehr unbeständige sein.

Ich habe früher gesagt, ich könne mir die Constitution der salzsauren Base nur in der frther erwähnten Weise denken; dies muss noch näher begründet werden, was ich hiemit versuchen will. 
Am deutlichsten spricht für die angegebene Constitution die Zersetzung des Körpers durch die Wärme. Als ich versuchte, das chlorwasserstoffsaure Salz der Base durch Destillation zu reinigen, trat vollständige Zersetzung ein.

Es entwickelte sich Formaldehyd, der sich theils durch seinen Geruch, theils durch seine Polymerisationsproducte nachweisen liess. In der Vorlage condensirte sich eine weisse, äusserst zerfliessliche Masse, die in Wasser gelöst wurde. $\mathrm{Zu}$ einem Theil der Lösung wurde überschüssige Sublimatlösung gesetzt, zu einem andern eine solche von Platinchlorid.

In beiden Fällen entstanden Niederschläge, die umkrystallisirt und analysirt wurden.

Hiebei bekam ich folgende Werthe:

a) Platindoppelsalz.

$0.4577 \mathrm{~g}$ Substanz gaben $0.1582 \mathrm{~g}$ Platin oder $34.56 \%$.

b) Quecksilberdoppelsalz.

$0 \cdot 3296 g$ Substanz gaben $0 \cdot 231 \mathrm{~g} \mathrm{Hg} \mathrm{S}$, entsprechend $0 \cdot 19913 \mathrm{~g} \mathrm{Hg}$ oder $60 \cdot 42 \%$.

Diese Werthe unterscheiden sich nur sehr wenig von denen, die den entsprechenden DoppeJsalzen des salzsauren Pyridins zukommen, denn diese verlangen:

a) $34 \cdot 27 \% \mathrm{Pt}$ und

b) $60 \cdot 83 \% \mathrm{Hg}$.

Es zerfällt demnach die salzsaure Base bei der Destillation in Formaldehyd und salzsaures Pyridin.

Bei der Oxydation der Base mit Kaliumpermanganat tritt eine ähnliche Zersetzung ein. Bringt man zur warmen Lösung der schwefelsauren Verbindung, der man überdies noch etwas Schwefelsäure zugesetzt hat, Kaliumpermanganat, so entwickelt sich unter starkem Aufbrausen Kohlensäure, und gleichzeitig tritt der charakteristische Geruch des Pyridins auf. Die salzsaure Base ist zu diesem Versuche minder gut geeignet, da sich der Kohlensäure dann immer etwas Chlor beimischt.

Diese Reactionen scheinen wobl die angeführte Constitutionsformel zu rechtfertigen. 
Diese auf vorstehend beschriebenem Wege erhaltene Base steht nicht vereinzelt da. Vor nicht allzulanger Zeit ist es nämlich dem italienischen Chemiker Coppola ${ }^{1}$ gelungen durch Einwirkung von Glykolchlorhydrin auf Pyridin eine Base zu erhalten, die er Pyridinhydroxyaethylenammonium nannte. Demnach wiirde dem von mir dargestellten Körper, der ans dem Methylenchlorhydrin, als welches man den Chlormethylalkohol auffassen kann, entsteht, der Name Pyridinbydroxymethylenammonium zukommen.

Zum Schlusse will ich noch ganz kurz erwähnen, dass Chinolin und Chlormethylalkohol in ganz ähnlicher Weise, nur minder heftig aufeinander einwirken.

Das Reactionsproduct ist eine violettroth gefärbte, zähe Masse, die sich in Wasser und Alkohol sehr leicht löst. Aus der alkoholischen Lösung lassen sich durch Äther Krystallnadeln ausfällen, die wahrscheinlich das chlorwasserstoffsaure Salz einer durch Anlagerung von Chlormethylalkohol an das Chinolin entstandenen Base sind.

Auch eine Reihe schön krystallisirender Doppelsalze mit Sublimat, Platinchlorid ete. lässt sich erhalten.

Die Zahl der Analysen, die bisher mit diesen Verbindungen ausgefuhrt wurden, ist aber noch zn gering, um mit Bestimmtheit einen Ausspruch in Betreff der Zusammensetzung des Körpers thun zu können, ebenso sind auch die ïbrigen Untersuchungen über denselben noch nicht abgeschlossen, so dass ein näheres Eingehen auf diesen Gegenstand einem späteren Berichte vorbehalten bleiben möge.

1 Gazz. chim. 15, 331. 